The Astrophysical Journal, 679:910-919, 2008 May 20

(C) 2008. The American Astronomical Society. All rights reserved. Printed in U.S.A.

\title{
A NOVEL TECHNIQUE TO INFER IONIC CHARGE STATES OF SOLAR ENERGETIC PARTICLES
}

\author{
L. S. Sollitt, ${ }^{1}$ E. C. Stone, ${ }^{2}$ R. A. Mewaldt, ${ }^{2}$ C. M. S. Cohen, ${ }^{2}$ A. C. Cummings, ${ }^{2}$ \\ R. A. Leske, ${ }^{2}$ M. E. Wiedenbeck, ${ }^{3}$ And T. T. von Rosenvinge ${ }^{4}$ \\ Received 2006 December 8; accepted 2008 January 28
}

\begin{abstract}
In some large solar energetic particle (SEP) events, the intensities of higher energy SEPs decay more rapidly than at lower energies. This energy dependence varies with particle species, as would be expected if the decay timescale depended on a rigidity-dependent diffusion mean free path. By comparing the decay timescales of carbon, nitrogen, oxygen, neon, magnesium, silicon, sulfur, and iron, mean charge states are inferred for these (and other) elements in three SEP events between 1997 and 2002 at energies between 10 and $200 \mathrm{MeV}$ nucleon $^{-1}$. In a fourth event, upper limits for the charge states are inferred. The charge states of many different particle species are all consistent with a single source temperature; in two events in 1997 and 2002, the best-fit temperature is much higher than that of the corona, which could imply a contribution from solar flare material. However, comparison with lower energy iron charge states for the 1997 event implies that the observed high-energy charge state could also be understood as the result of stripping during shock acceleration in the corona.
\end{abstract}

Subject headings: acceleration of particles — methods: data analysis — Sun: particle emission

\section{INTRODUCTION}

Two of the open questions in space physics are where solar energetic particles (SEPs) come from and how they are accelerated. If there are no nonthermal charge-changing processes during particle acceleration, the charge states of the SEPs accelerated from a solar plasma will be representative of the conditions at the source. In gradual events, where particles are accelerated out of the corona by a shock driven by a coronal mass ejection, one would expect that particles would have charge states consistent with a temperature of $1-2 \mathrm{MK}$ for a coronal acceleration site; in impulsive events, which are thought to occur at solar flare sites, the temperature would be up to $10 \mathrm{MK}$ (Reames 1999). Calculations of expected equilibrium charge states as a function of temperature for various astrophysically abundant elements are given in Arnaud \& Rothenflug (1985) and Arnaud \& Raymond (1992) and more recently in Mazzotta et al. (1998).

Recently, various authors (Ostryakov \& Stovpyuk 1999; Barghouty \& Mewaldt 2000; Kovaltsov et al. 2001; Lytova \& Kocharov 2005) have suggested that there might be processes during acceleration that could render the observed charge state different from its thermal value. This might include ionization and recombination due to collisions with electrons and other ions in the source plasma. Given a long enough period of time or a dense enough plasma (or a large enough product of the density and acceleration time), a particle population at a given energy will assume an equilibrium charge distribution, whose average charge state increases with energy, just as ions in an accelerator beam do when they are passed through a foil. A typical product of acceleration time and density might be on the order of $10^{9} \mathrm{~s} \mathrm{~cm}^{-3}$ (Ostryakov \& Stovpyuk 1999; Kovaltsov et al. 2001). It is possible that high- $Z$ particles such as silicon and iron might not be in a dense enough plasma for a long enough period of time for the population average charge state to reach its equi-

\footnotetext{
${ }^{1}$ Northrop Grumman Space Technology, R1/1132D 1 Space Park, Redondo Beach, CA 90278; luke.sollitt@ngc.com.

2 Division of Physics, Mathematics, and Astronomy, 220-47, California Institute of Technology, Pasadena, CA 91125.

3 Jet Propulsion Laboratory, Pasadena, CA 91109.

${ }^{4}$ NASA/Goddard Space Flight Center, Code 661, Greenbelt, MD 20771.
}

librium value. Higher energy particles, spending longer times at the acceleration site, might have charge states closer to the equilibrium value than at lower energies. Lower $Z$ particles such as oxygen might become fully stripped in the acceleration process.

A possible indication of a nonthermal charge-changing process in solar particle acceleration would be an average charge state for a high- $Z$ species, such as iron, that increases with energy. The challenge is to obtain an average charge state at high energies. There is no method to directly measure ionic charge states of energetic particles at high energy $\left(\sim 10-100 \mathrm{MeV}\right.$ nucleon $\left.^{-1}\right)$ with existing spacecraft instrumentation. Indirect methods that have been used previously include the geomagnetic cutoff technique using data from the SAMPEX spacecraft (Leske et al. 1995; Mazur et al. 1999; Labrador et al. 2003), the abundance fractionation method of Cohen et al. (1999), and the rise time method of Dietrich \& Tylka (2001) and Dietrich \& Lopate (1999). In this work, a new method to infer average charge states at high energy is presented. Charge states are inferred (or limits given) in four events; comparison is made to charge states at lower energies for one of these events. A more complete treatment of this work is given in Sollitt (2004).

\section{MODEL AND METHOD}

Large gradual SEP events generally follow similar time intensity profiles: an onset whose rate will depend on how well connected the event is magnetically to the Earth, followed by a slow ( days) exponential or quasi-exponential decay, as was first observed before the space age in a ground-level event by Meyer et al. (1956). Figure 1 shows time intensity profiles of iron ions for the 1997 November 6 solar particle event. The particle intensities plotted in Figure 1 are $3 \mathrm{hr}$ averages at various energies as measured with the Solar Isotope Spectrometer (SIS; Stone et al. 1998) on board the Advanced Composition Explorer spacecraft. The uncertainties shown are purely statistical. Iron particle intensities rise by $2-3$ orders of magnitude over the course of a few hours during this event but then decay away exponentially over the course of a few days. In the figure it can be seen that iron intensities decay away more rapidly at higher energies: there is an energy dependence (or rigidity dependence) to the decay timescale. 


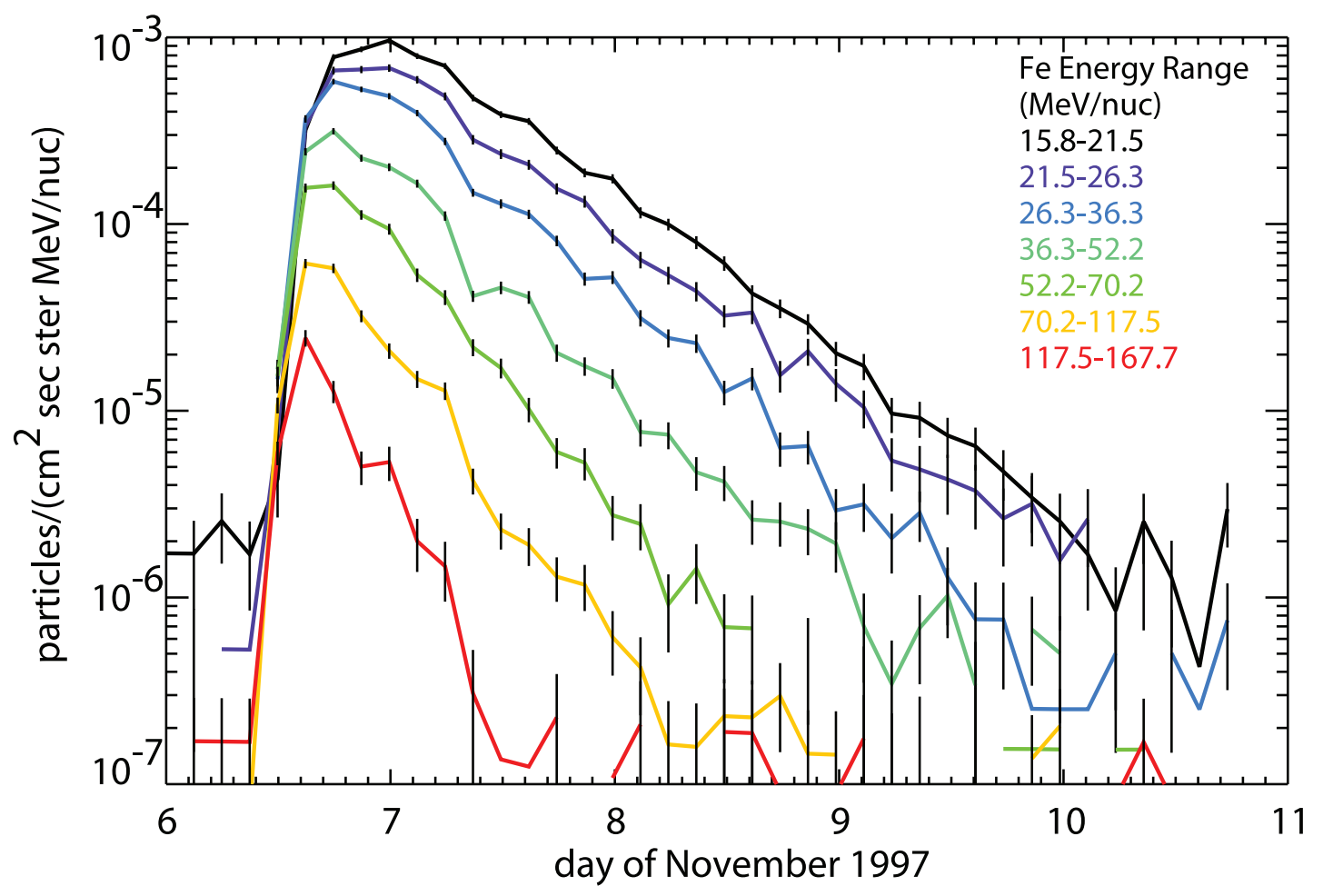

FIG. 1.-Fluxes of iron at various energies in the 1997 November 6 solar particle event. As energy increases the flux decays away more rapidly.

The inference of average charge states for SEP species will arise from the characteristic decay lifetimes for each species as a function of energy. The characteristic decay lifetime for a given particle population will depend on its rigidity: on its momentum and charge to mass ratio, or stated differently, on its energy and average charge state. For this reason, what we seek to understand is the decay timescale for SEPs as a function of energy and species.

Early theoretical efforts, such as that in Meyer et al. (1956), described a solar particle event as an impulsive injection, followed by diffusion away from the inner heliosphere through a medium. This medium might be a thick shell, a thin shell, or be in a number of other configurations. As the years have gone by, refinements have been made to this model, with such additions as a diffusion cavity that extends from the Sun past $1 \mathrm{AU}$ with an outer boundary, convection in the solar wind, and adiabatic cooling (Parker 1963; Burlaga 1967; Forman 1971; Lupton 1973; Lupton \& Stone 1973). All of these authors solve a Fokker-Planck equation for SEP particle density $n$, sometimes called the Parker equation:

$\frac{\partial n}{\partial t}=\nabla \cdot(\underline{\underline{\kappa}} \cdot \nabla n)-\nabla \cdot(n \boldsymbol{V})+\frac{1}{3} \nabla \cdot \boldsymbol{V}\left\{\frac{\partial}{\partial T}[\alpha(T) T n]\right\}$.

Here $\kappa$ is the diffusion coefficient; $V$ is the solar wind speed, assumed to be radial; $T$ is the particle kinetic energy; and $\alpha(T)=$ $\left(T+2 M_{0} c^{2}\right) /\left(T+M_{0} c^{2}\right)$. In this equation, the terms on the right are the diffusive term, the convection term, and the adiabatic energy loss term.

A full description of the method by which charge states are inferred is given in Appendix A. A basic discussion will be given here. Different solutions arise from assumptions about the diffusion coefficient and nondiffusive effects. The solution of Burlaga (1967) is for the case where convection and adiabatic cooling are ignored. The solution of Forman (1971) assumes a diffusion coefficient that is linear with distance from the Sun inside the diffusion cavity. The solution of Lupton (1973) and Lupton \& Stone
(1973) assumes a diffusion coefficient that is constant with respect to distance from the Sun. Figure 2 shows model calculations of decay timescales as a function of energy for the three solutions of the Parker equation for a single charge-to-mass ratio. For this figure, the inverse of the decay timescale is plotted. One can see that at high values of the diffusion coefficient, the decay rate ( $1 /$ decay timescale) is a power law in the diffusion coefficient, and therefore in rigidity and energy. At lower values of the diffusion coefficient, the decay timescale (or rate) is approximately constant. This seems to be true for both the case where the diffusion coefficient is constant with heliocentric radius and the case where it is linear. Although these solutions are spherically symmetric, this suggests a parameterization of the form (see Appendix A)

$$
\frac{1}{\tau}=\frac{1}{\tau_{C}}+W(\alpha E)^{\gamma}
$$

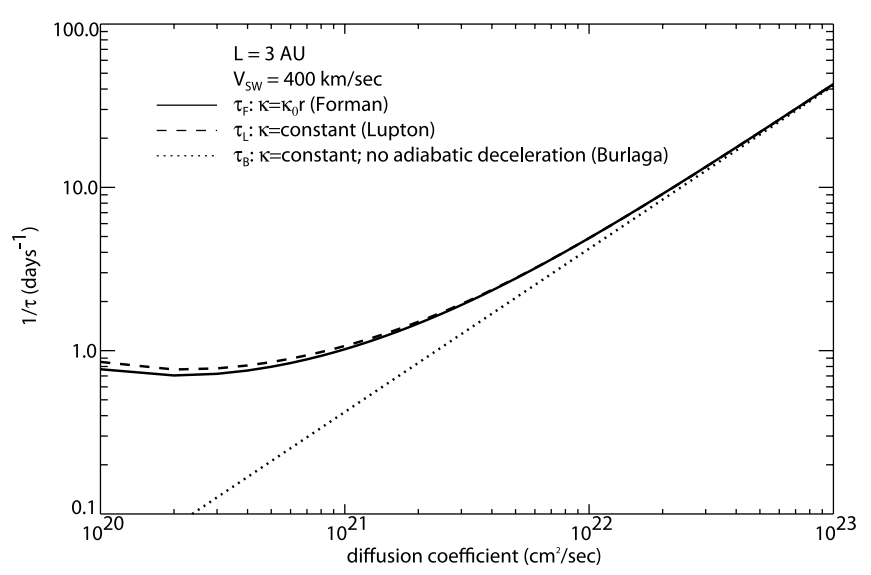

FIG. 2.-Model calculations of decay times as a function of the diffusion coefficient for the three solutions of the Parker equation discussed. 

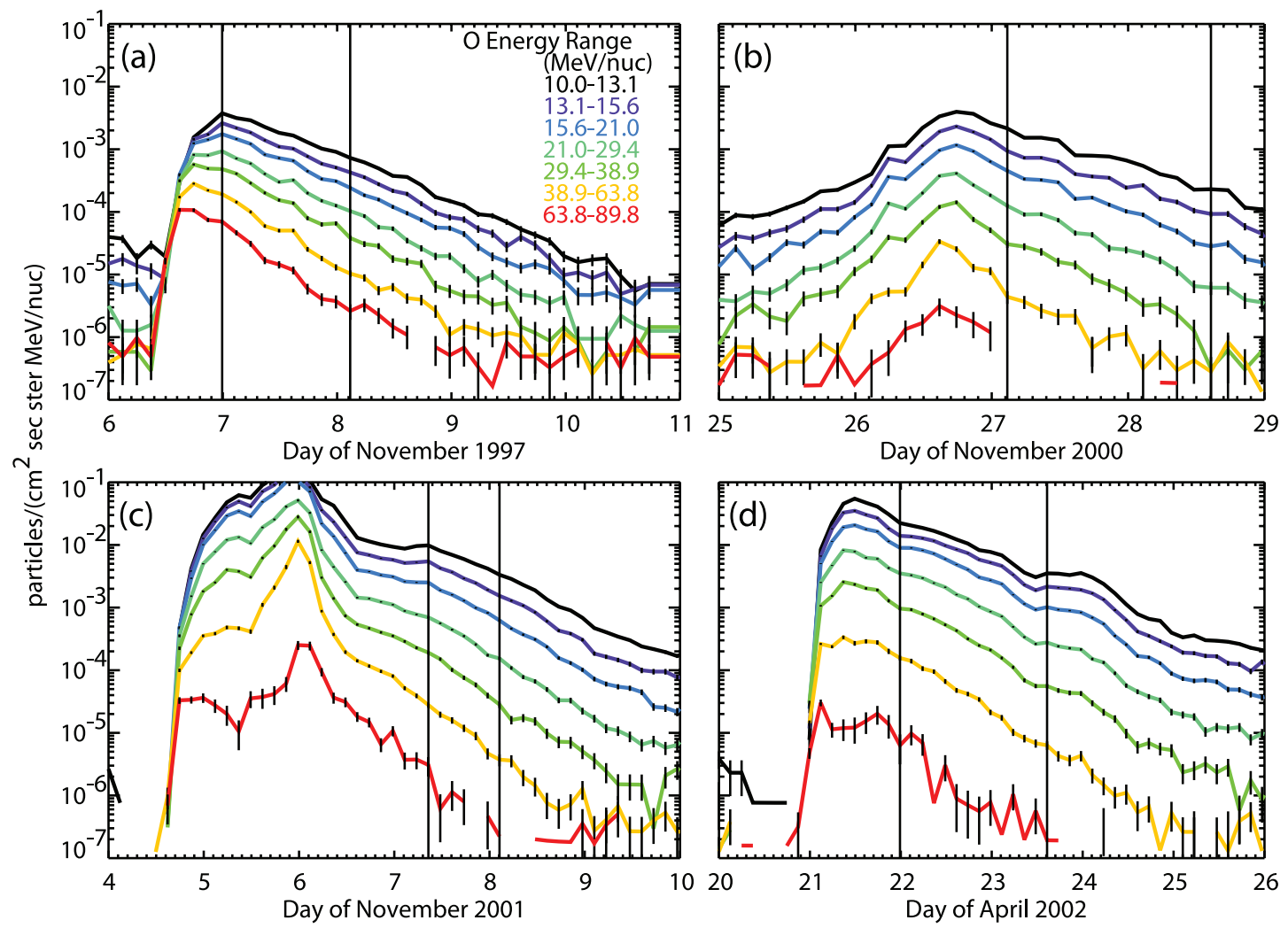

Fig. 3.-Fluxes of oxygen at various energies in the four solar particle events analyzed here. As energy increases the flux decays away more rapidly.

Here $\tau_{C}$ is the effective constant term of the decay timescale at lower energies (low values of the diffusion coefficient); $\gamma$ is the power-law index of the energy for the high-energy (high values of the diffusion coefficient) relation. There are two constants of proportionality for the power law: $W$ is an overall coefficient that applies to the entire system; $\alpha$ is different for each particle species and is referenced to a species whose charge state is taken to be understood; in this work, we have chosen carbon to be the reference species. For a given species $X$ (of atomic mass $A_{X}$ ) and a reference species $Y$ (of atomic mass $A_{Y}$ and ionic charge $Q_{Y}$ ), the charge state $Q_{X}$ will be given by (see Appendix A)

$$
Q_{X}=\alpha^{\gamma /(1-2 \gamma)} \frac{Q_{Y}}{A_{Y}} A_{X} .
$$

In order to find an average charge state, one fits equation (2) to at least one species $X$ and a reference species $Y$ with $\tau_{C}, \gamma, W$, and $\alpha$ as free parameters; the charge state is then calculated from equation (3). In practice, several different species $X$ will be included in a fit, each with a different free parameter $\alpha_{X}$.

In order to test the validity of the parameterization, values of $1 / \tau$ as a function of energy per nucleon were calculated for both the $\kappa=\kappa_{0} r$ and $\kappa=$ constant cases. Those values of $1 / \tau$ were then used as pseudodata to be fitted (for a single species) with equation (2), setting $\alpha=1$. For both $\kappa=\kappa_{0} r$ and $\kappa=$ constant, the fractional deviation between the exact solution and the fit parameterization function for $E>7 \mathrm{MeV}$ nucleon $^{-1}$ was found to be less than half a percent. For $E>10 \mathrm{MeV}$ nucleon $^{-1}$, the deviation was less than $0.1 \%$. Note that in the limit $\tau_{C} \rightarrow \infty$, the solution of Burlaga (1967) is described exactly by the parameterization. Therefore, when fitting data to the parameterization, the result of a finite $\tau_{C}$ will denote a system where adiabatic deceleration is important.
Four solar particle events were analyzed in this work: events starting on 1997 November 6, 2000 November 26, 2001 November 4 , and 2002 April 21. Figure 3 shows sets of time intensity profiles for the solar particle events analyzed in this work. The intensities plotted in Figure 3 are $3 \mathrm{hr}$ averages for oxygen ions at various energies as measured with the SIS instrument. As with Figure 1, the uncertainties shown are purely statistical.

Data from the Ultra Low Energy Isotope Spectrometer (ULEIS; Mason et al. 1998), also aboard $A C E$, were included in three of the events analyzed (G. M. Mason 2007, personal communication) in order to better understand $\tau_{C}$. In the 1997 November 6 event, carbon was included at $1.81-2.56 \mathrm{MeV}_{\text {nucleon }}{ }^{-1}$, and oxygen was included in the energy ranges $1.28-1.81,1.81-2.56$, and $3.62-5.12 \mathrm{MeV}$ nucleon $^{-1}$. In the 2001 November 4 event, oxygen was included at 2.56-5.12 MeV nucleon ${ }^{-1}$ (which would be a combination of the 2.56-3.62 and 3.62-5.12 $\mathrm{MeV}$ nucleon $^{-1}$ energy ranges). In the 2002 April 21 event, oxygen was used at the same energy range.

In each SEP event, observations were restricted to those occurring during a period of interest. During this period of interest, particle intensities are seen to decay exponentially with time: these are periods of equilibrium decay. The vertical lines in each panel of Figure 3 denote the beginning and end of the period of interest for each SEP event. Before each period of interest, other physical processes such as new injections or passing shocks can dominate the particle intensity profile. The end of the period of interest is chosen either to avoid further injections or local particle acceleration, as in the case of the 2002 April event, or because higher energy or less abundant fluxes have decayed below the background. For each SEP event, a single common period of interest was used for all particle species. A full discussion of the period selection process is given in Sollitt (2004). 


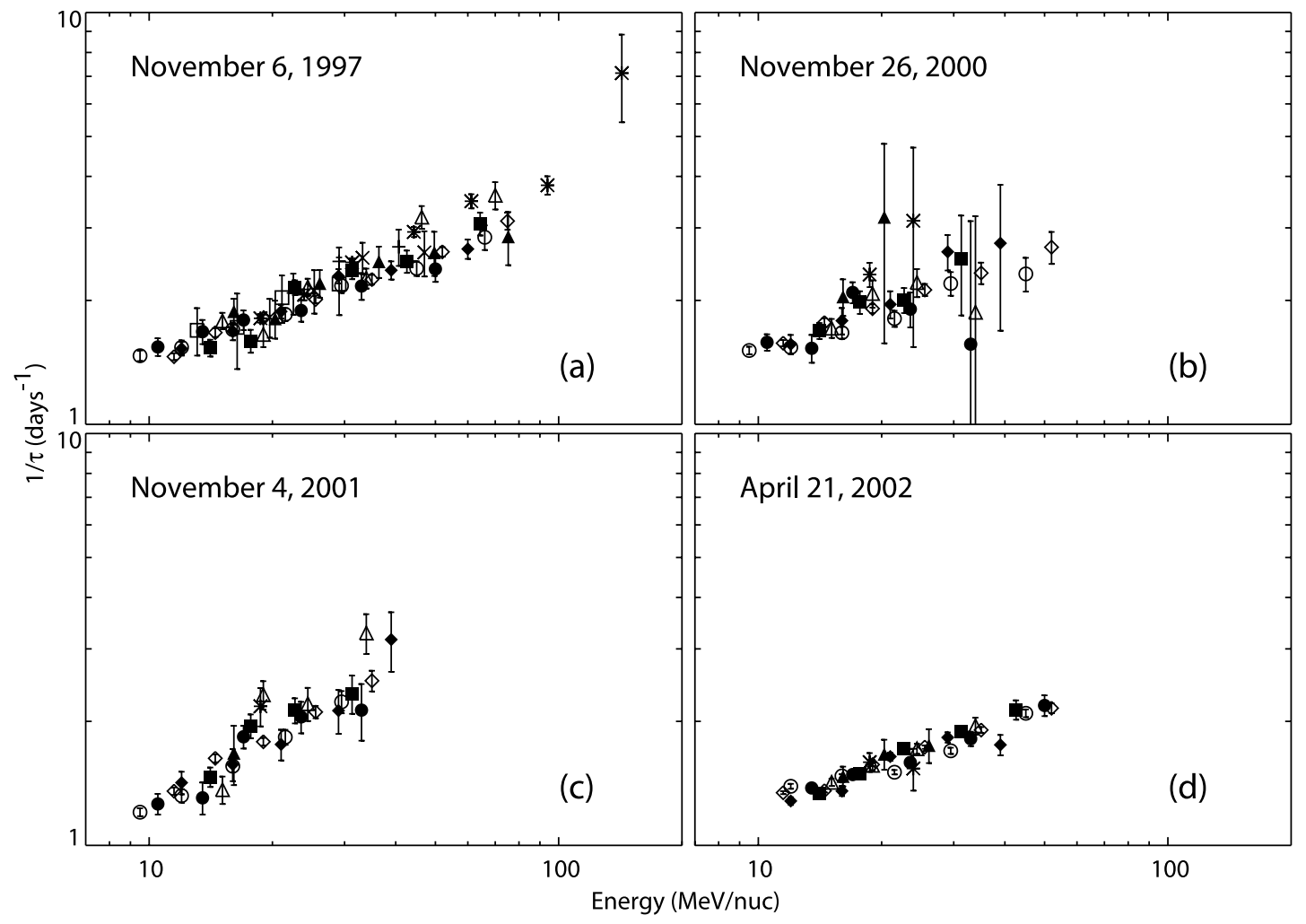

FIG. 4.-Decay rates for all of the elements and the four events analyzed. Analogous to Fig. $5 a$. The particle species shown are carbon (open circles), nitrogen ( filled circles), oxygen (open diamonds), neon ( filled diamonds), sodium (open squares), magnesium ( filled squares), silicon (open triangles), sulfur ( filled triangles), calcium (crosses), iron (asterisks), and nickel $(X)$. Panel $a$ shows the 1997 November 6 event; panel $b$ shows the 2000 November 26 event; panel $c$ shows the 2001 November 4 event; and panel $d$ shows the 2002 April 21 event.

Maximum likelihood fits were done using Poisson statistics, as least-squares fitting with Gaussian statistics was seen to introduce systematic deviations in the decay rates (Sollitt 2004). For all of the profiles examined, the fit function used was an exponential decay; for decay profiles in which the counts dropped below two particles in a $3 \mathrm{hr}$ period, a constant background was included in the fit. Figure 4 shows the fitted decay rates of all of the elements used in each of the events analyzed.

Figure $5 a$, shows plots of the decay rate, $1 / \tau$, versus energy per nucleon for carbon (open circles), oxygen ( filled diamonds), and iron ( filled squares). These data are for the 1997 November 6 solar particle event. It is evident that all of these species follow the same kind of curve, of the sort predicted by equation (A20).
Carbon and oxygen follow the same curve, which would indicate that they have very similar charge-to-mass ratios. Note that the iron points do not lie atop the carbon and oxygen points. It is apparent that iron must have a charge-to-mass ratio different from those of oxygen and carbon. Panel $b$ shows the same data, only now the energies of the three different species have been adjusted by the various $\alpha_{X}$ from the fit. The solid line represents the decay rate versus energy curve as calculated using the global fit parameters $\tau_{C}, W$, and $\gamma$. Recall that for carbon, $\alpha_{\mathrm{C}}=1$ is fixed. Another way to picture the fit would be to imagine a series of curves, each with the same values of global fit parameters, but multiplied by the various values $\alpha_{X}$. For the elements displayed in Figure 5 we have $\alpha_{\mathrm{C}}=1, \alpha_{\mathrm{O}}=0.995_{-0.023}^{+0.024}$, and $\alpha_{\mathrm{Fe}}=1.277_{-0.035}^{+0.036}$.

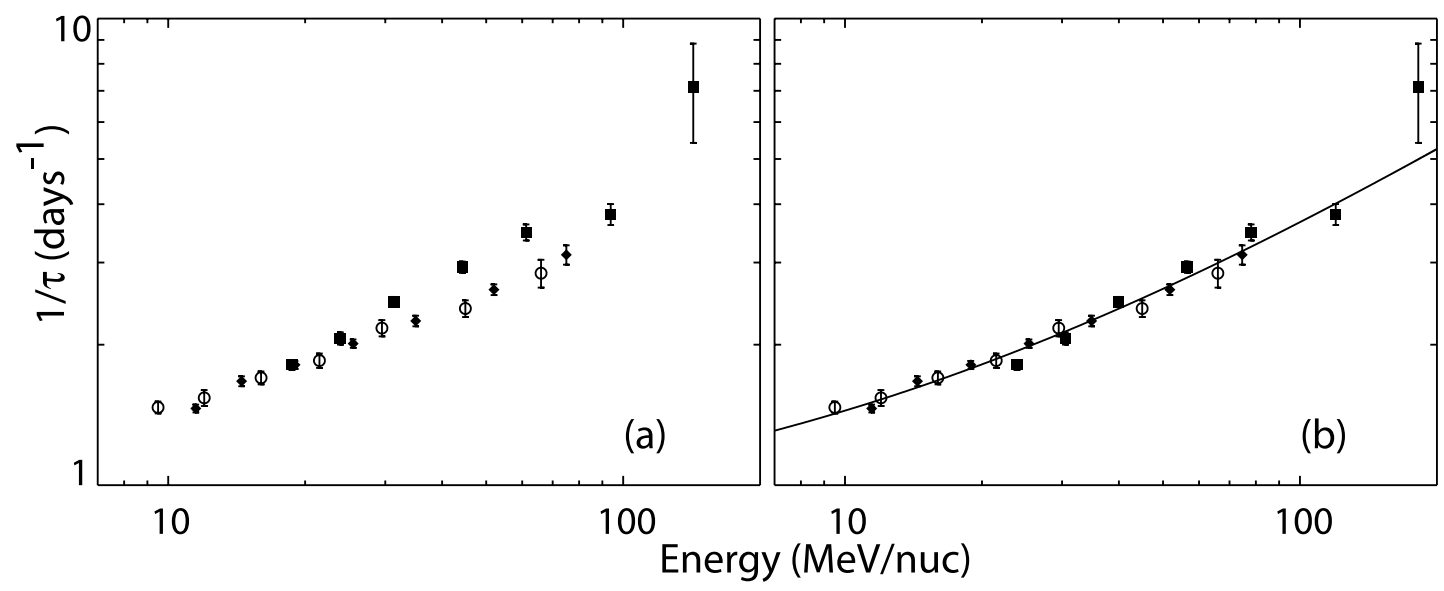

FIG. 5.-Decay rate vs. energy (in MeV nucleon ${ }^{-1}$ ) for carbon (open circles), oxygen ( filled diamonds), and iron ( filled squares) in the 1997 November 6 event. Panel $a$ shows the decay rates prior to fitting with eq. (2); panel $b$ shows the global fit (solid line) and adjusts the energies for oxygen and iron by their respective $\alpha_{X}$. 
TABLE 1

Mean Charge States Inferred for the Various Events Analyzed, along with the Overall Fit Parameters

\begin{tabular}{|c|c|c|c|c|}
\hline Element & 1997 Nov 6 & 2000 Nov 26 & 2001 Nov 4 & 2002 Apr 21 \\
\hline Nitrogen .......... & $6.9 \pm 1.1$ & $\leq 7.0$ & $6.47 \pm 0.68$ & $6.75_{-0.65}^{+0.66}$ \\
\hline Oxygen.............. & $7.95_{-0.44}^{+0.46}$ & $\leq 6.3$ & $6.83_{-0.62}^{+0.51}$ & $6.76_{-0.68}^{+0.69}$ \\
\hline Neon.................. & $9.3 \pm 1.0$ & $\leq 7.6$ & $9.4 \pm 1.1$ & $10.13_{-0.92}^{+0.96}$ \\
\hline Sodium ............. & $7.4_{-3.6}^{+3.8}$ & $\ldots$ & $\ldots$ & $\ldots$ \\
\hline Magnesium...... & $9.8 \pm 1.4$ & $\leq 9.3$ & $9.9_{-1.5}^{+1.3}$ & $10.3 \pm 1.2$ \\
\hline Silicon ............. & $8.8_{-1.8}^{+1.7}$ & $\leq 8.7$ & $10.9_{-2.0}^{+1.3}$ & $11.3_{-1.8}^{+1.9}$ \\
\hline Sulfur.... & $11.9 \pm 2.9$ & $\ldots$ & $\begin{array}{r}13.0_{-5.9}^{+6.0} \\
\end{array}$ & $11.8_{-4.1}^{+4.8}$ \\
\hline Calcium ............ & $9.1_{-3.7}^{+3.6}$ & $\ldots$ & $\ldots$ & $\ldots$ \\
\hline Iron ...................... & $15.3_{-3.3}^{+3.0}$ & $\ldots$ & $14.2_{-6.9}^{+6.0}$ & $21.6_{-8.0}^{+8.4}$ \\
\hline Nickel ............... & $19.6_{-6.5}^{+6.6}$ & $\ldots$ & $\ldots$ & $\ldots$ \\
\hline $1 / \tau_{C} \ldots \ldots \ldots \ldots \ldots$ & $0.771_{-0129}^{+0.098}$ & $0.485_{-1.343}^{+0.438}$ & $0.416_{-0.506}^{+0.228}$ & $0.756_{-0.165}^{+0.119}$ \\
\hline$W \ldots \ldots$ & $0.157_{-0.036}^{+0.055}$ & $0.266_{-0.204}^{+1.059}$ & $0.147_{-0.075}^{+0.248}$ & $0.118_{-0.046}^{+0.079}$ \\
\hline$\gamma \ldots$. & $0.633_{-0.060}^{+0.054}$ & $0.492_{-0.274}^{+0.332}$ & $0.740_{-0.217}^{+0.169}$ & $0.632_{-0.105}^{+0.107}$ \\
\hline
\end{tabular}

The analysis of Mason et al. (2006) uses comparison factors $\alpha_{X}$ of the same form used here, although derived from examination of the entire profile of an event, rather than from decay timescales. This treatment must account for the onset phase of the event, where the profile is dominated by transport inside $1 \mathrm{AU}$. By contrast, the work here is focused on periods that are dominated by transport outside $1 \mathrm{AU}$.

\section{RESULTS}

Table 1 gives all the charge states calculated from the various fit factors $\alpha_{X}$ with accompanying uncertainties, along with the overall fit parameters $W, 1 / \tau_{C}$, and $\gamma$. Table 2 shows the energy ranges for these inferred average charge states. In each event a charge state for carbon of $Q_{\mathrm{C}}=5.9$ was assumed. This charge state is consistent with a wide variety of source locations for carbon, including solar flare sites and the corona. For the 2000 November 26 event, the best-fit value for $\gamma$ is very close to 0.5 , which would indicate a diffusion coefficient that was independent of rigidity. The uncertainty is quite large, however, and the upper bound on $\gamma$ has been used to deduce upper limits for the charge states in this event. Uncertainties are not presented for the charge states in this event. For the other events, the uncertainties $\sigma_{Q_{X}}$ are calculated from the uncertainties $\sigma_{\alpha_{X}}$ in using standard error propagation with equation (A19):

$$
\sigma_{Q_{X}}^{2}=\sigma_{\alpha_{X}}^{2}\left[\left(\frac{\gamma}{1-2 \gamma}\right) \frac{Q_{X}}{\alpha_{X}}\right]^{2}+\sigma_{\gamma}^{2}\left[Q_{X} \frac{\ln \alpha_{X}}{(1-2 \gamma)^{2}}\right]^{2} .
$$

For some of the elements and events in Table 1, the dominant term in the uncertainty $\sigma_{Q_{X}}$ will derive from $\sigma_{\alpha_{X}}$. For others, such as iron in the 1997 November event, the dominant source of uncertainty will be in $\sigma_{\gamma}$.

Figure 6 shows inferred charge states (filled circles) for four solar particle events. Panel $a$ shows the 1997 November 6 event; panel $b$ shows upper limits for the 2000 November event; panel $c$ shows the 2001 November 4 event; and panel $d$ shows the 2002 April 21 event. Also shown are best-fit temperatures with $1 \sigma$ limits, for the assumption that there are no nonthermal chargechanging processes. The temperatures were fit using linear interpolations of the calculations of Mazzotta et al. (1998).

For comparison with the current work, the results of Cohen et al. (1999; squares) and Mazur et al. (1999; diamonds) are included in Figure $6 a$; the results of Labrador et al. (2003; diamonds) are included in Figures $6 c$ and $6 d$. All of these results are at energies comparable to the ones here. There are no results with which to compare for the 2000 November 26 event. It can be seen in Figure $6 a$ that the results of Cohen et al. (1999), inferring charge states from abundances, are largely consistent with the current work. In Figures $6 c$ and $6 d$, it can be seen that the results of Labrador et al. (2003) are also consistent with the current work, although there does seem to be a small systematic difference. However, in the 1997 November 6 event, as shown in Figure $6 a$, the results of Mazur et al. (1999), who also used the geomagnetic cutoff technique, are systematically higher. This discrepancy is an indication of the challenges in inferring charge states at high energy.

Table 3 shows the best-fit temperatures for source plasmas in the four events from Figure 6. In the case of the 2000 November 26 event, the temperature is an upper limit. It may be reasonably postulated that for the 2000 November 26 and 2001 November 4 events, the source plasma is of coronal origin, as the best-fit temperatures are consistent with temperatures in the corona, which typically are around 1-2 MK (Bochsler 2000). For the 1997 November 6 event and the 2002 April 21 event, however, the best-fit temperatures are higher than is typical for the corona. Measurements like these have led to the suggestion that nonthermal charge-changing processes occur during SEP acceleration (Kovaltsov et al. 2001).

Calculations for charge states at energies measured in SIS have been done by Reames et al. (1999), Ostryakov \& Stovpyuk (1999), Barghouty \& Mewaldt (2000), Kovaltsov et al. (2001), and Lytova \& Kocharov (2005; see also Kocharov 2006). In particular, Kovaltsov et al. (2001) present detailed calculations of the expected charge state of iron as a function of energy for various different values of parameters in the acceleration process.

TABLE 2

Energy Ranges for the Inferred Charge States in Table 1

\begin{tabular}{|c|c|c|c|c|}
\hline Element & $\begin{array}{c}1997 \text { Nov } 6 \\
\text { (in } \mathrm{MeV} \text { nucleon }^{-1} \text { ) }\end{array}$ & $\begin{array}{c}2000 \text { Nov } 26 \\
\text { (in } \mathrm{MeV} \text { nucleon }^{-1} \text { ) }\end{array}$ & $\begin{array}{c}2001 \text { Nov } 4 \\
\text { (in } \mathrm{MeV} \text { nucleon }^{-1} \text { ) }\end{array}$ & 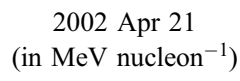 \\
\hline 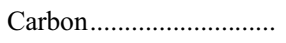 & $8.62-76.34$ & $8.62-54.30$ & $8.62-33.21$ & $11.23-54.30$ \\
\hline 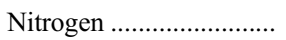 & $9.33-59.19$ & $9.33-36.17$ & $9.33-36.17$ & $12.18-59.19$ \\
\hline 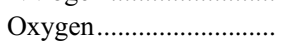 & $9.99-89.78$ & $9.99-89.78$ & $9.99-38.94$ & $13.07-63.77$ \\
\hline 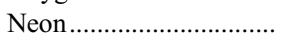 & $11.15-72.24$ & $11.15-72.24$ & $11.15-44.02$ & $11.15-44.02$ \\
\hline 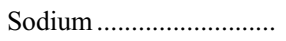 & $11.34-34.08$ & & $\ldots$ & $\ldots$ \\
\hline Magnesium.......................... & $12.16-79.97$ & $12.16-36.64$ & $12.16-36.64$ & $12.16-48.63$ \\
\hline Silicon & $13.04-87.14$ & $13.04-39.76$ & $13.04-39.76$ & $13.04-39.76$ \\
\hline 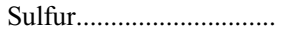 & $13.83-93.85$ & $\ldots$ & $13.83-18.37$ & $13.83-30.10$ \\
\hline Calcium ............................... & $20.30-47.87$ & $\ldots$ & $\ldots$ & $\ldots$ \\
\hline Iron & $15.83-167.66$ & $\ldots$ & $15.83-21.53$ & $15.83-26.30$ \\
\hline Nickel ................................. & $16.71-55.47$ & $\ldots$ & $\ldots$ & $\ldots$ \\
\hline
\end{tabular}




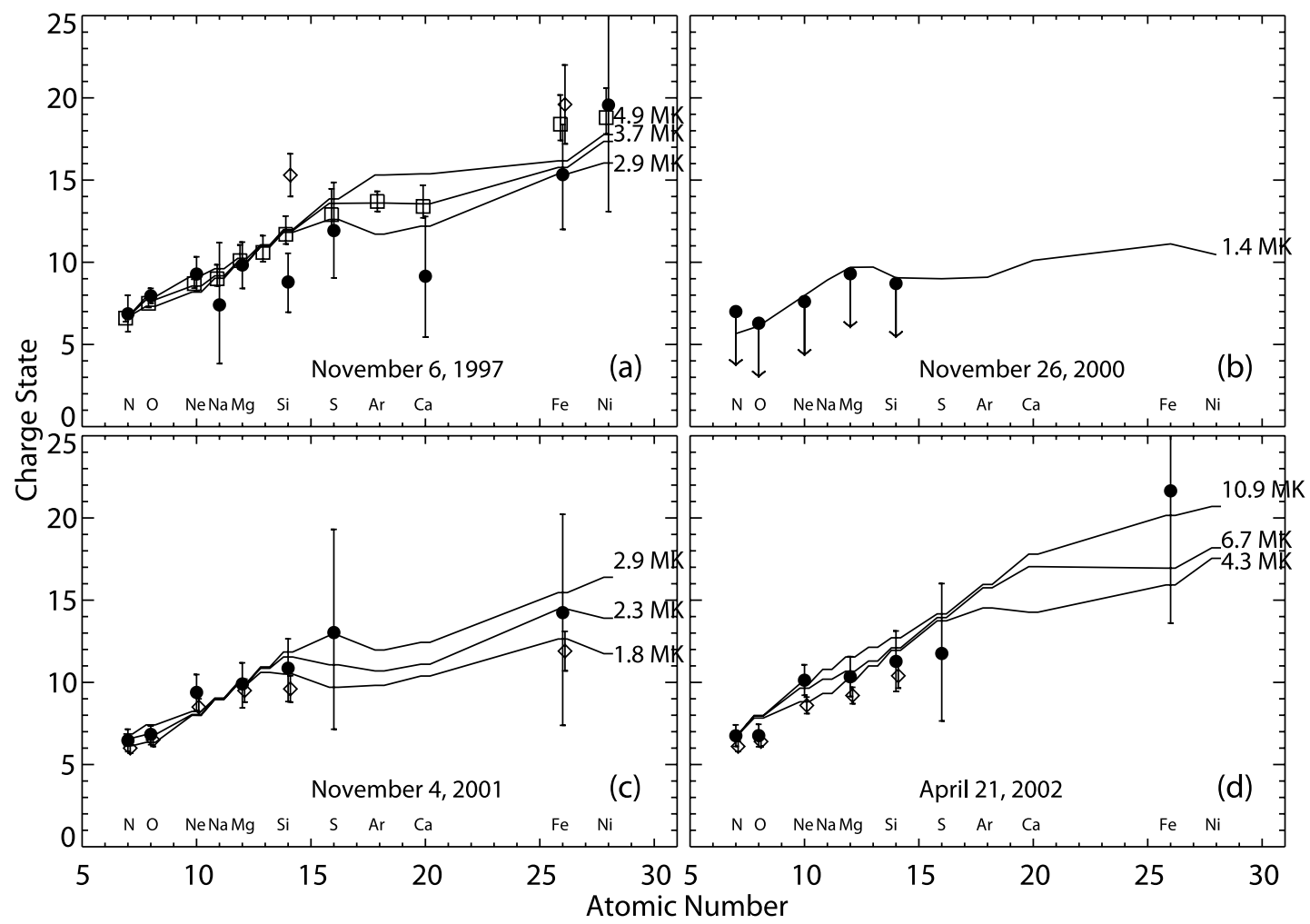

FIG. 6. - Charge states in four solar particle events analyzed. Filled circles represent the current work. In panel $a$, open diamonds represent the results of Mazur et al. (1999), and open squares represent the results from Cohen et al. (1999). In panels $c$ and $d$, open diamonds represent the results of Labrador et al. (2003). In each panel, the center solid line represents the best interpolated fit temperature; the upper and lower solid lines are the $1 \sigma$ upper and lower limit temperatures.

In modeling the acceleration mechanism Kovaltsov et al. (2001) assumed that the rate of energy gain in acceleration of a particle would be a power law in the particle's energy

$$
\left(\frac{d E}{d t}\right)_{a}=\frac{E_{1}}{\tau_{1}}\left(\frac{E}{E_{1}}\right)^{S}
$$

where $E_{1}$ is taken to be $1 \mathrm{MeV}$ nucleon $^{-1}$ and $\tau_{1}$ is the time required to accelerate the particle to that energy. In making their calculations, several values of the power-law index $S$ were examined. The charge state energy dependence for a given event will be determined by $S$ and the product of $\tau_{1}$ and the density $n$ (in particles per $\mathrm{cm}^{3}$ ) at the particle acceleration site.

Figure 7 shows charge state as a function of energy for iron calculated by Kovaltsov et al. (2001) for different values of $n \times \tau_{1}$ and $S$. For these calculations, the charge state at $30 \mathrm{MeV}$ nucleon ${ }^{-1}$ has been fixed at $Q_{\mathrm{Fe}}=18$. The solid line is for $S=1$ and $n \times \tau_{1}=2 \times 10^{9} \mathrm{~cm}^{-3} \mathrm{~s}$ (the "equilibrium condition" of Kovaltsov et al. 2001). The dashed line is for $S=0.5$ and $n \times \tau_{1}=7.5 \times$ $10^{8} \mathrm{~cm}^{-3} \mathrm{~s}$ (the "nonequilibrium condition" of Kovaltsov et al. 2001). Either of these curves might represent the high-energy

TABLE 3

Fit Temperatures for the Four Solar Particle Events

\begin{tabular}{|c|c|}
\hline Event & $\begin{array}{c}\text { Temperature } \\
\text { (MK) }\end{array}$ \\
\hline 1997 Nov $6 . . .$. & $3.7_{-0.8}^{+1.2}$ \\
\hline 2000 Nov 26 & $\leq 1.4$ \\
\hline 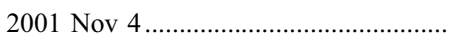 & $2.3_{-0.5}^{+0.6}$ \\
\hline 2002 Apr $21 \ldots \ldots \ldots \ldots \ldots \ldots \ldots \ldots \ldots \ldots$ & $6.7_{-2.4}^{-4.2}$ \\
\hline
\end{tabular}

data; a different selection of the parameters would also fit either of the sets of low-energy data as well.

The various calculations of charge stripping processes in shock acceleration can be made to describe the energy dependence of particle charge states in the 1997 November 6, event reasonably well. The temperatures assumed in these models are all typical for the corona. In this event, the energy dependence of the mean charge state of iron indicates that the temperature shown in Table 3 is not a true source temperature. If one is seeing charge stripping

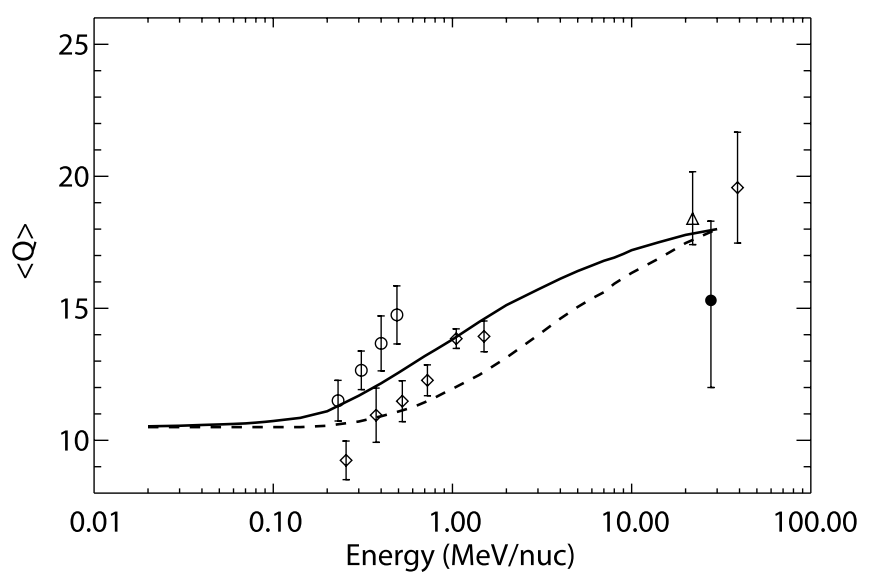

FIG. 7.- Mean charge state of iron as a function of energy as calculated by Kovaltsov et al. (2001). Here the iron charge state at $30 \mathrm{MeV}$ nucleon $^{-1}$ has been fixed at a value of $Q_{\mathrm{Fe}}=18$, and calculations have been done for $n \times \tau_{1}=2 \times$ $10^{9} \mathrm{~cm}^{-3} \mathrm{~s}$ and $S=1$ (solid curve) and for $n \times \tau_{1}=7.5 \times 10^{8} \mathrm{~cm}^{-3} \mathrm{~s}$ and $S=0.5$ (dashed curve). Also plotted are the various results for the 1997 November 6 event: the current result (solid circle), the results of Möbius et al. (1999; open circles) and Mazur et al. (1999; open diamonds), and the Cohen et al. (1999; open triangle) result. 
in this event, then the overall effect is to strip multiple particle species such that they all serendipitously appear to have arisen from a higher temperature source than the corona, within our limited statistics.

\section{CONCLUSIONS}

The propagation model used to deduce charge states in this work is practically as old as the field of space physics itself. Newer models have extended it for the periods of event onsets. In the equilibrium decay phase of a solar particle event it is still quite accurate. The preceding pages have shown that this model can be used to infer average charge states of solar energetic particles in a relatively straightforward way. In solar particle events with appropriate conditions, that is, an equilibrium decay phase, one can deduce the mean charge states of solar particles at energies of $\sim 10-100 \mathrm{MeV}$ nucleon $^{-1}$, which would be a very challenging measurement to make directly.

The mean charge states deduced here have been used to examine two different and competing ideas on the origins of solar energetic particles. In one, solar energetic particles are accelerated without stripping out of plasma with which they are in thermal equilibrium. In this case, the observed charge states are indicative of conditions in the source plasma. In the other model, particles are accelerated out of the corona, and stripping occurs. The observed charge states are indicative of the acceleration time and density of the corona where the acceleration occurs.

The 2000 November 26 and 2001 November 4 events.- For these two events, the inferred mean charge states (and the resulting inferred temperatures) are consistent with acceleration from a coronal source plasma. In the case of the 2000 November 26 event, only upper limits for mean charge states and the source temperature could be inferred, but these upper limits are also consistent with acceleration from the corona. This implies that little stripping is occurring during acceleration in these events.
The 1997 November 6 event.- When looking at charge state data assembled from a number of sources in the 1997 November 6 event, it becomes apparent that the temperature fitted here to this event cannot represent the temperature of a single source plasma: otherwise, one would find the same charge states at low energies as one finds at higher energies. Instead, iron seems to show an increasing charge state with increasing energy. These data would be consistent with the suggestion of mixing between a low-energy coronal source population and a high-energy impulsive flare population, as has been suggested by several authors (Mason et al. 1999; Cohen et al. 1999; Mazur et al. 1999; Möbius et al. 1999; Cane et al. 2003, 2006), and as could be described by the model of Tylka et al. (2005) and Tylka \& Lee (2006).

Alternatively, the recent work in modeling charge equilibration from stripping in shock acceleration in a dense plasma also gives results that compare favorably with the data for the 1997 November 6 event. In comparing the model calculations of Kovaltsov et al. (2001) with iron, the observed charge state energy dependence of these elements can be adequately described by the model. This model shows that the data are consistent with acceleration in this event taking place in a plasma at coronal temperatures.

The 2002 April 21 event. - The temperature inferred for the 2002 April 21 event may also not be a true temperature, but be indicative of this kind of charge-changing process. Extending the model of Kovaltsov et al. (2001) to other elements might allow a calculation of expected mean charge state due to stripping versus atomic number at the energies observed in SIS. Armed with such a calculation, measurements of the sort done here at high energies might possibly be used as a probe of the acceleration process even when lower energy measurements of charge states are unavailable.

This work was supported by NASA at Caltech, JPL, and GSFC under grants NAG5-6912 and NAG5-12929.

\section{APPENDIX A}

Forman (1971) solves the Fokker-Planck equation for SEP diffusion (the Parker equation) for a diffusion coefficient $\kappa$ that is proportional with heliocentric radius: $\kappa=\kappa_{0} r$, where $\kappa_{0}$ is a constant. For the equilibrium decay phase of a solar particle event, the characteristic decay time $\tau_{\mathrm{F}}$ found by Forman (1971) depends on the diffusion coefficient $\kappa$ in the following way:

$$
\tau_{\mathrm{F}}=\frac{4 L}{\left(j_{\eta, 1}\right)^{2} \kappa_{0}}
$$

where $L$ is the boundary of the diffusive cavity, $j_{\eta, 1}$ is the first nonzero value of a dimensionless quantity $x$ where the Bessel function $J_{\eta}(x)$ goes to zero, and $\eta$ is given by

$$
\eta=2\left[\left(1-\frac{V}{2 \kappa_{0}}\right)^{2}+\frac{2 C V}{\kappa_{0}}\right]^{1 / 2}
$$

Here $V$ is the solar wind speed and $C$ is the Compton-Getting factor, given by

$$
C=\frac{2+2 \delta}{3}
$$

for the case where adiabatic deceleration is the only energy loss mechanism and the differential particle flux $j(E)$, sometimes written as $d J / d E$, is given by $j(E) \sim E^{-\delta}$. In this and other simple solutions to the Parker equation, the boundary $L$ is taken to be a discrete location beyond which the diffusion constant becomes large. No particles diffuse back from beyond this boundary. This is only an approximation, although Pioneer and Voyager observations (Webber et al. 1979) have shown that there is a transition region in space beyond which the diffusion coefficient is much larger than in the inner heliosphere. 
In a different solution where $\kappa=$ constant is assumed (Lupton 1973; Lupton \& Stone 1973) the corresponding decay timescale $\tau_{\mathrm{L}}$ is given by

$$
\tau_{\mathrm{L}}=\frac{\kappa}{4 \kappa^{2} \psi+V^{2}}
$$

where once again, $V$ is the solar wind speed, and $\psi$ solves the boundary condition

$$
F_{0}\left(\frac{\beta}{2 \sqrt{\psi}}, \sqrt{\psi} L\right)=0
$$

where $F_{0}$ is a Coulomb wave function (a form of confluent hypergeometric function), and $\beta$ (which here is not particle speed) is given by

$$
\beta=\frac{V(2 C-1)}{\kappa} .
$$

A third and earlier solution by Burlaga (1967) incorporated diffusion only. The terms of the Fokker-Planck equation associated with the solar wind speed, that is, convection and adiabatic cooling, were neglected. In this solution, one finds the decay timescale $\tau_{\mathrm{B}}$ to be given by

$$
\tau_{\mathrm{B}}=\frac{L^{2}}{\pi^{2} \kappa}
$$

Figure 2 shows calculations of the decay rate, $1 / \tau$, for the three different solutions of the Parker equation as a function of the diffusion coefficient $\kappa$. For these calculations, a boundary of $3 \mathrm{AU}$ was used, and the solar wind speed was assumed to be $400 \mathrm{~km} \mathrm{~s}^{-1}$. The SEP spectral index $\delta$ was assumed to be 3. As can be seen in Figure 2, the decay timescales $\tau_{\mathrm{L}}$ and $\tau_{\mathrm{F}}$ are very similar at all values of $\kappa$.

For $\tau_{\mathrm{F}}$ at large $\kappa$, one can see from equation (A2) that the terms in $\eta$ involving $\kappa$ become small and $\eta$ tends to a value of 2 . Thus, the $j_{\eta, 1}$ turn into $j_{2,1}$, and the decay time is inversely proportional to the diffusion coefficient. Examining the large $\kappa$ behavior of $\tau_{\mathrm{L}}$, it can be seen from equation (A6) that at large $\kappa, \beta$ tends to zero. Thus, from equation (A5), $\psi$ becomes independent of the diffusion coefficient. For large enough $\kappa$, the solar wind speed $V$ can be neglected in equation (A4) (the $\kappa$ term dominates), and once again, the decay time will be inversely proportional to the diffusion coefficient. It can be seen in Figure 2 that at large $\kappa$, both $\tau_{\mathrm{F}}$ and $\tau_{\mathrm{L}}$ converge to the value $\tau_{\mathrm{B}}$ that was derived by Burlaga (1967), neglecting convection and adiabatic deceleration. Indeed, one finds that at large $\kappa, \psi$ is inversely proportional to $L^{2}$. At large $\kappa$ (and for $V \rightarrow 0$ ), one finds

$$
\psi=\frac{\pi^{2}}{4 L^{2}} .
$$

At smaller $\kappa, \tau_{\mathrm{F}}$ and $\tau_{\mathrm{L}}$ diverge from $\tau_{\mathrm{B}}$ as effects due to convection and adiabatic cooling become important. At intermediate $\kappa\left(\sim 10^{20}\right.$ $\left.10^{21} \mathrm{~cm}^{2} \mathrm{~s}^{-1}\right)$ both $\tau_{\mathrm{F}}$ and $\tau_{\mathrm{L}}$ have little dependence on $\kappa$, and at low $\kappa\left(<10^{20} \mathrm{~cm}^{2} \mathrm{~s}^{-1}\right)$ decrease with $\kappa$. This is consistent with observations at intermediate energies $\left(\sim 1 \mathrm{MeV}\right.$ nucleon $\left.{ }^{-1}\right)$ of time and space independent particle spectra having decay times that are independent of energy and species (Reames et al. 1997; McKibben 1972). Then at intermediate $\kappa$, both $\tau_{\mathrm{F}}$ and $\tau_{\mathrm{L}}$ are dominated by a decay timescale $\tau_{C}$ due to convection and cooling.

The inclusion of the factors $\eta$ and $\psi$ in the two solutions of the Parker equation makes understanding decay timescales in terms of those solutions very complicated, perhaps more complicated than is strictly necessary. Both solutions tend to behave the same way at intermediate to high energies (and $\kappa$ ), suggesting a simple parameterization. In effect, at intermediate to high energies (energies relevant to SEPs) there are two superposed decays for the flux $j$ : a decay timescale $\tau_{C}$ that is constant with energy and particle species (or velocity and rigidity) that dominates at intermediate energies, and a decay timescale $\tau_{D}$ that depends on the diffusion coefficient and dominates at higher energies. This suggests the following parameterization:

$$
j=D e^{-t / \tau_{C}} e^{-t / \tau_{D}(\kappa)}=D e^{-t / \tau} .
$$

From this, one can see that the decay timescale of a particle population will be given by

$$
\frac{1}{\tau}=\frac{1}{\tau_{C}}+\frac{1}{\tau_{D}(\kappa)} .
$$

This is a much simpler expression mathematically than either equation (A1) or equation (A3) above because only the (much simpler) limiting forms of the decay constant, $\tau_{C}$ and $\tau_{D}(\kappa)$, are used.

Dröge (1994) shows that the mean free path $\lambda$ for SEP diffusion scales as a power law in the rigidity $R$ :

$$
\lambda=\lambda_{0} R^{\gamma_{0}}=\lambda_{0} R^{0.2-0.4} .
$$

The normalization constant $\lambda_{0}$ and the power-law index $\gamma_{0}$ depend on various parameters of the solar event, including the magnetic field strength, the solar wind speed, and the power spectrum of the interplanetary magnetic turbulence. The range of $0.2-0.4$ represents 
typical values for $\gamma_{0}$ as derived by Dröge (1994) from energetic electron and ion data. In a multispacecraft study during two solar events in November and December of 1977, Beeck et al. (1987) measured rigidity dependences of $\lambda \sim R^{0.45}$ and $\sim R^{0.2}$, respectively.

From this one deduces the velocity and rigidity dependence of the diffusion coefficient:

$$
\kappa \equiv \frac{1}{3} v \lambda=\frac{1}{3} v \lambda_{0} R^{\gamma_{0}} .
$$

Then the large- $\kappa$ decay timescale $\tau_{D}$ will be given by

$$
\tau_{D}=\frac{3 W_{1}}{v \lambda}=\frac{1}{W_{0} v R^{\gamma_{0}}} .
$$

Here $W_{1}$ is the constant of proportionality between $\kappa$ and $\tau_{D}$; it will have different values for each solution to the Parker equation. The constant $W_{0}$ folds in the constant $\lambda_{0}$. In fitting the data it will be allowed to float. This suggests an expression for $\tau_{D}$ that parameterizes the various solutions of the Parker equation for SEP propagation:

$$
\frac{1}{\tau}=\frac{1}{\tau_{C}}+\frac{1}{\tau_{D}(\kappa)}=\frac{1}{\tau_{C}}+W_{0} v R^{\gamma_{0}} .
$$

Folding all of the constants into a new constant $W$, one is left with an expression that includes only the quantities that are of interest: the average SEP atomic mass $A$, the average ionic charge $Q, \gamma_{0}$, and the energy per nucleon $E_{\mathrm{KE} / n}$ [recalling that for these nonrelativistic energies $\left.E=m v^{2} / 2=\frac{1}{2}\left(Q^{2} / m\right) R^{2}\right]$ :

$$
\frac{1}{\tau}=\frac{1}{\tau_{C}}+W\left(\frac{A}{Q}\right)^{\gamma_{0}} E_{\mathrm{KE} / n}^{\left(\gamma_{0}+1\right) / 2}=\frac{1}{\tau_{C}}+W\left(\frac{A}{Q}\right)^{2 \gamma-1} E_{\mathrm{KE} / n}^{\gamma} .
$$

Here the new power-law index $\gamma=\left(\gamma_{0}+1\right) / 2$ is the actual quantity that will be fit to the data.

Equation (A15) does not actually determine, by itself, the charge state $Q$ of a particle species. There is a degeneracy in the free parameters $W, Q$, and $\gamma$. The constant $W$ is treated as a free parameter in the system, since it depends on the power spectrum of the interplanetary magnetic turbulence (and on $L$ ), which varies from event to event as shown from fitting particle time intensity profiles and anisotropies (Dröge 2000a, 2000b). In examining the measured decay timescales for various particle species in a solar event, the power-law index $\gamma$ is also allowed to float, and $Q$ is unknown. They all combine in a degenerate way: $W$ is multiplied by $Q$, which is raised to a power of $\gamma$. However, what happens when two particle species $X$ and $Y$ have the same decay timescale? Since the quantities $\tau_{C}, \gamma$, and $W$ are the same for both species, it can be seen from equation (A15) that

$$
\left(\frac{A_{X}}{Q_{X}}\right)^{2 \gamma-1} E_{(\mathrm{KE} / n)_{X}}^{\gamma}=\left(\frac{A_{Y}}{Q_{Y}}\right)^{2 \gamma-1} E_{(\mathrm{KE} / n)_{Y}}^{\gamma} .
$$

Both $E_{(\mathrm{KE} / n)_{X}}$ and $E_{(\mathrm{KE} / n)_{Y}}$ are measured. Then we have

$$
E_{(\mathrm{KE} / n)_{Y}}=\left(\frac{Q_{Y}}{A_{Y}} \frac{A_{X}}{Q_{X}}\right)^{(2 \gamma-1) / \gamma} E_{(\mathrm{KE} / n)_{X}}=\alpha E_{(\mathrm{KE} / n)_{X}},
$$

where a multiplicative constant is now defined:

$$
\alpha \equiv\left(\frac{Q_{Y}}{A_{Y}} \frac{A_{X}}{Q_{X}}\right)^{(2 \gamma-1) / \gamma} .
$$

If the mean charge state of species $Y$ is known, then the mean charge state of species $X$ can be found by determining the multiplicative constant, $\alpha$, that separates the energies (in MeV nucleon ${ }^{-1}$ ) of the particle species at the same decay timescale $\tau$. Then the charge state of species $X$ is found by solving equation (A18):

$$
Q_{X}=\alpha^{\gamma /(1-2 \gamma)} \frac{Q_{Y}}{A_{Y}} A_{X} .
$$

In applying this parameterization to the data, the inverses of the decay lifetimes for each species are fitted to the following equation:

$$
\frac{1}{\tau}=\frac{1}{\tau_{C}}+W(\alpha E)^{\gamma} .
$$

The values of $\tau_{C}, \gamma$, and $W$ are all allowed to float but are constrained to be the same for all species. For each species, a separate $\alpha_{X}$ is allowed to float. In this analysis the reference element used is carbon. For carbon, $\alpha_{\mathrm{C}}=1$ is assumed. In order to account for the mass distributions in SEPs, an average mass for each particle species is used; this average mass is calculated from the abundances of nuclides presented in Anders \& Grevesse (1989). 


\section{REFERENCES}

Anders, E., \& Grevesse, N. 1989, Geochim. Cosmochim. Acta, 53, 197 Arnaud, M., \& Raymond, J. 1992, ApJ, 398, 394

Arnaud, M., \& Rothenflug, R. 1985, A\&AS, 60, 425

Barghouty, A. F., \& Mewaldt, R. A. 2000, in AIP Conf. Proc. 528, Acceleration and Transport of Energetic Particles Observed in the Heliosphere, ed. R. A. Mewaldt et al. (Melville: AIP), 71

Beeck, J., Mason, G. M., Hamilton, D. C., Wibberenz, G., Kunow, H., Hovestadt, D., \& Klecker, B. 1987, ApJ, 322, 1052

Bochsler, P. 2000, Rev. Geophys., 38, 247

Burlaga, L. F. 1967, J. Geophys. Res., 72, 4449

Cane, H. V., Mewaldt, R. A., Cohen, C. M. S., \& von Rosenvinge, T. T. 2006, J. Geophys. Res., 111, A06590, DOI: 10.1029/2005JA011071

Cane, H. V., von Rosenvinge, T. T., Cohen, C. M. S., \& Mewaldt, R. A. 2003, Geophys. Res. Lett., 30(12), 8017

Cohen, C. M. S., et al. 1999, Geophys. Res. Lett., 26, 149

Dietrich, W., \& Lopate, C. 1999, in Proc. 26th Int. Cosmic Ray Conf. (Melville), 6,91

Dietrich, W., \& Tylka, A. 2001, in Proc. 27th Int. Cosmic Ray Conf. (Hamburg), 27,3173

Dröge, W. 1994, ApJS, 90, 567

2000a, Space Sci. Rev., 93, 121

2000b, ApJ, 537, 1073

Forman, M. A. 1971, J. Geophys. Res., 76, 759

Kocharov, L. 2006, in Modeling the Energy-Dependent Charge States of Solar Energetic Particles (AGU Geophys. Mon. Ser. 165; Washington, DC: AGU), 137

Kovaltsov, G. A., Barghouty, A. F., Kocharov, L., Ostryakov, V. M., \& Torsti, J. 2001, A\&A, 375, 1075

Labrador, A., Leske, R., Mewaldt, R., Stone, E., \& von Rosenvinge, T. 2003, in Proc. 28th Int. Cosmic Ray Conf. (Tsukuba), 6, 3269
Leske, R. A., Cummings, J. R., Mewaldt, R. A., Stone, E. C., \& von Rosenvinge, T. T. 1995, ApJ, 452, L149

Lupton, J. E. 1973, Ph.D. thesis, California Institute of Technology

Lupton, J. E., \& Stone, E. 1973, J. Geophys. Res., 78, 1007

Lytova, M., \& Kocharov, L. 2005, ApJ, 620, L55

Mason, G. M., Desai, M. I., Cohen, C. M. S., Mewaldt, R. A., Stone, E. C., \& Dwyer, J. R. 2006, ApJ, 647, L65

Mason, G. M., et al. 1998, Space Sci. Rev., 86, 409 1999, Geophys. Res. Lett., 26, 141

Mazur, J. E., Mason, G. M., Looper, M. D., Leske, R. A., \& Mewaldt, R. A. 1999, Geophys. Res. Lett., 26, 173

Mazzotta, P., Mazzitelli, G., Colafrancesco, S., \& Vittorio, N. 1998, A\&AS, 133,403

McKibben, R. B. 1972, J. Geophys. Res., 77, 3957

Meyer, P., Parker, E. N., \& Simpson, J. A. 1956, Phys. Rev., 104, 768

Möbius, E. et al. 1999, Geophys. Res. Lett., 26, 145

Ostryakov, V. M., \& Stovpyuk, M. F. 1999, Sol. Phys., 189, 357

Parker, E. N. 1963, Interplanetary Dynamical Processes (New York: Interscience) Reames, D. V. 1999, Space Sci. Rev., 90, 413

Reames, D. V., Kahler, S. W., \& Ng, C. K. 1997, ApJ, 491, 414

Reames, D. V., Ng, C. K., \& Tylka, A. J. 1999, Geophys. Rev. Lett., 26, 3585

Sollitt, L. 2004, Ph.D. thesis, California Institute of Technology

Stone, E. C., et al. 1998, Space Sci. Rev., 86, 357

Tylka, A. J., Cohen, C. M. S., Dietrich, W. F., Lee, M. A., Maclennan, C. G., Mewaldt, R. A., Ng, C. K., \& Reames, D. V. 2005, ApJ, 625, 474

Tylka, A. J., \& Lee, M. A. 2006, ApJ, 646, 1319

Webber, W. R., McDonald, F. B., Trainor, J. H., \& von Rosenvinge, T. T. 1979 in Proc. 16th Int. Cosmic Ray Conf. (Midori-cho Tanashi), 5, 353 